\title{
Philosophiques
}

\section{Étapes vers un monde sans schizophrénie}

\section{Jeffrey Poland}

Volume 33, numéro 1, printemps 2006

Philosophie et psychopathologie

URI : https://id.erudit.org/iderudit/012949ar

DOI : https://doi.org/10.7202/012949ar

Aller au sommaire du numéro

Éditeur(s)

Société de philosophie du Québec

ISSN

0316-2923 (imprimé)

1492-1391 (numérique)

Découvrir la revue

Citer cet article

Poland, J. (2006). Étapes vers un monde sans schizophrénie. Philosophiques, 33(1), 99-124. https://doi.org/10.7202/012949ar
Résumé de l'article

Cet article présente des arguments selon lesquels le concept de schizophrénie est un stéréotype préjudiciable qu'il vaudrait mieux éliminer de la recherche et de la pratique clinique. Les obstacles à une telle élimination sont repérés, et nous discutons des stratégies pour les surmonter. d'utilisation que vous pouvez consulter en ligne.

https://apropos.erudit.org/fr/usagers/politique-dutilisation/ 


\title{
Étapes vers un monde sans schizophrénie
}

\author{
JEFFREY POLAND \\ Université de Brandeis \\ poland@brandeis.edu
}

\begin{abstract}
RÉSUMÉ. - Cet article présente des arguments selon lesquels le concept de schizophrénie est un stéréotype préjudiciable qu'il vaudrait mieux éliminer de la recherche et de la pratique clinique. Les obstacles à une telle élimination sont repérés, et nous discutons des stratégies pour les surmonter.
\end{abstract}

\begin{abstract}
The paper presents arguments for the claim that the concept of schizophrenia is a harmful stereotype that is best eliminated from research and clinical practice. Obstacles to such elimination are identified and strategies for overcoming them are discussed.
\end{abstract}

\section{Introduction}

Pendant plus d'un siècle, le concept de schizophrénie, ou son prédécesseur celui de dementia praecox, a occupé une place centrale dans les conceptualisations cliniques et scientifiques de certaines formes de maladie mentale grave. Les cliniciens diagnostiquent régulièrement chez les individus un trouble mental nommé "schizophrénie ", pour lequel il existe une variété de stratégies et de techniques cliniques. Les scientifiques poursuivent toujours des recherches qui portent sur l'épidémiologie, l'étiologie, la pathologie, et la phénoménologie clinique (c'est-à-dire, les expressions cliniques et la dynamique caractéristique) de la schizophrénie. Naturellement, l'entraînement professionnel de tels cliniciens et chercheurs comprend des connaissances et des pratiques en rapport avec la schizophrénie. À tous les niveaux d'éducation formelle, les manuels de psychiatrie et de psychologie anormale comportent des chapitres consacrés à la schizophrénie, dans lesquels les critères diagnostiques, les modèles théoriques, les techniques de recherche, les découvertes empiriques et les manières d'intervenir cliniquement sont présentés de façon systématique. L'éducation informelle des patients, de leur famille et de leurs amis, des administrateurs, de ceux qui sont en charge d'élaborer des politiques, des médias et du public en général souligne et incorpore souvent des connaissances concernant la schizophrénie. Évidemment, les modèles de financement et les politiques sociales qui ont trait aux maladies mentales formulent (entre autres) celles-ci en termes de schizophrénie, laquelle est conçue comme un trouble mental grave et débilitant qui pose un problème majeur de santé publique et doit être résolu. Ainsi, la schizophrénie est un concept qui joue un rôle organisationnel significatif dans un grand nombre de pratiques cliniques, scientifiques, et sociales. Il structure de façon envahissante la conscience et la conceptualisation de ce qui constitue une maladie mentale grave dans toutes les sphères de la société. 
Au cœur de ces pratiques et conceptualisations, il existe quelque chose comme une « idée reçue " qui sert de fondation à toute pensée et action concernant la schizophrénie ; cette idée reçue comprend des affirmations comme :

1) - La schizophrénie est une maladie cérébrale ${ }^{1}$. On ne connaît pas tous les détails de sa pathologie et de son étiologie;

- La schizophrénie a des taux de prévalence stables à travers le temps et les cultures (par exemple, approximativement $1 \%$ de prévalence); - L'étiologie de la schizophrénie, comme on l'a établi, indique que cette maladie a une composante génétique (comme en témoignent, par exemple, des études sur la famille, les jumeaux et l'adoption) ${ }^{2}$;

- La schizophrénie est associée à de nombreux facteurs environnementaux qui peuvent jouer un rôle dans son étiologie (par exemple, l'exposition prénatale à la famine, l'infection virale et le stress ; le traumatisme à la naissance) ;

- La recherche dans le domaine de la pathophysiologie de la schizophrénie a fourni plusieurs résultats intéressants et des pistes prometteuses (par exemple la dérégulation dopaminergique, la ventriculomégalie, l'hypofrontalité, l'hypertemporalité, les déficits neurocognitifs);

- L'étiologie de la schizophrénie est donc conçue comme un cadre diathèse-stress dans lequel la vulnérabilité génétique interagit avec des facteurs environnementaux pour déclencher, éventuellement, la maladie ; - Un soutien empirique en faveur d'un modèle neurodévelopmental de la schizophrénie ${ }^{3}$ existe déjà ;

2) La schizophrénie a un profil clinique caractéristique et identifiable (par exemple, une présentation clinique, un début et une évolution caractéristique) et peut être diagnostiquée de façon fiable à l'aide des critères du DSM-IV (voir annexe).

3) La schizophrénie a des conséquences préjudiciables sur le plan psychologique et social, et constitue un sérieux problème de santé publique.

4) Le traitement de base pour la schizophrénie consiste en l'utilisation de drogues psychotropiques. Ce traitement s'est amélioré de façon importante dans la dernière décennie (par exemple, par le biais des anti-psychotiques atypiques). La schizophrénie est gérée (atténuation, soutien,

1. La schizophrénie a souvent été vue comme une maladie spectrale qui présente une variation relativement continue dans les motifs [patterns] de sa pathologie, dans son étiologie et dans ses manifestations cliniques. Néanmoins, son statut de maladie n'est pas remis en cause dans les rangs des tenants de l'idée perçue, et on croit invariablement qu'il existe quelque chose comme une pathologie de base qui constitue l'essence de la maladie et qui présente une certaine quantité de variations contingentes.

2. Le modèle génétique qui est actuellement préféré est le modèle polygénique, multifactoriel et interactif ; voir Gottesman, 1991, 1994 ; Moldin et Gottesman, 1997.

3. Voir Hirsh et Weinberger, 2003 pour des textes représentatifs concernant l'étiologie et la pathologie de la schizophrénie. 
prévention de rechute, réhabilitation) grâce à une combinaison de traitements pharmacologiques primaires et de techniques secondaires appropriées (par exemple, des interventions psychosociales) ${ }^{4}$.

5) La schizophrénie est stigmatisée de façon injustifiée, et cette stigmatisation peut être réduite en enseignant que la schizophrénie est une maladie cérébrale.

De telles affirmations contribuent à soutenir l'idée que la schizophrénie existe et qu'elle est une véritable maladie mentale ${ }^{5}$, que l'on en sait beaucoup à son sujet (mais pas tout), qu'il y a des programmes de recherche productifs et prometteurs sur des questions importantes à son sujet (c'est-à-dire, que c'est un sujet digne de recherche empirique) et que les pratiques cliniques concernant la schizophrénie ont des bases scientifiques et sont de plus en plus efficaces. Selon l'idée reçue, la recherche sur la schizophrénie est un domaine scientifique important et prometteur qui mérite des ressources humaines considérables et les millions de dollars de recherche qui y sont consacrés. De plus, la crédibilité de cette recherche ainsi que l'utilité de ses découvertes garantissent les rôles importants que le concept de schizophrénie et les affirmations qui en font usage jouent dans les politiques sociales, l'administration des soins de santé, l'éducation, la science et la pratique clinique : c'est-à-dire, le fait que le concept de schizophrénie occupe un rôle culturel significatif dépend essentiellement de la crédibilité scientifique et pragmatique de l'idée reçue.

La critique du concept de schizophrénie et des affirmations et pratiques qui y sont associées a pris deux formes générales : sociopolitique et scientifique. Tandis que le premier type de critique tente de mettre au jour les engagements idéologiques et les intérêts que sert le concept ainsi que d'évaluer son impact social, le deuxième type de critique conteste directement sa crédibilité scientifique et son utilité pratique, et donc la légitimité du rôle qu'il joue sur le plan clinique, social et en ce qui concerne la recherche. Notons que, jusqu'à un certain point, les critiques individuelles ${ }^{6}$ mêlent habituellement les deux sortes de critiques, la distinction entre critique socio-politique et critique scientifique servant principalement à identifier divers types de stratégies critiques. Les critiques individuelles orientent habituellement leurs analyses vers l'un ou l'autre de ces types. Même si les deux types de critiques ont mené à des idées valables et ont permis de cerner les sujets de discussion qui méritent une attention sérieuse, la critique en général n'a pas encore réussi à mettre sérieusement à l'épreuve

4. Voir McEvoy et al., 1999, pour des directives représentatives des pratiques cliniques liées au traitement de la schizophrénie.

5. Dans la psychiatrie contemporaine, affirmer qu'une condition est un trouble mental, c'est comme affirmer qu'elle est une "dysfonction préjudiciable " (harmful dysfunction) telle que caractérisée de façon imparfaite par le DSM, ou peut-être plus adéquatement par Wakefield (1992).

6. Parmi les critiques importants qui ont précisément été impliqués dans les deux genres de stratégies, on compte : Bentall (1990), Boyle (2002), Szasz (1988), Laing (1967), Scheff (1999), et Sarbin (1990). 
l'idée reçue sur la schizophrénie et à diminuer son impact. Autrement dit, malgré des décennies de critique serrée, des millions de dollars sont encore dépensés en recherches sur la schizophrénie. Les pratiques cliniques tournent encore de manière importante autour du concept de schizophrénie, quelques chapitres des manuels scolaires présentent encore de l'information sur la schizophrénie et les politiques sociales sont encore élaborées autour de l'idée d'une supposée maladie cérébrale appelée "schizophrénie ".

De nombreuses questions émergent de ce contexte et méritent d'être discutées. L'idée reçue sur la schizophrénie est-elle crédible du point de vue scientifique ? Y a-t-il une justification pragmatique à la prépondérance de l'idée reçue ? Je pense que la réponse à chacune de ces questions est très clairement « non ». En fait, en supposant que l'idée reçue manque à la fois de crédibilité scientifique et d'utilité pragmatique, une question supplémentaire se pose : que doit-on faire pour effectuer une réforme significative des conceptualisations et des pratiques courantes concernant les maladies mentales graves ?

Mon plan dans ce texte est le suivant. Dans la première partie, je proposerai de bonnes raisons de penser que l'idée reçue sur la schizophrénie n'est pas crédible scientifiquement. Dans la seconde partie, j'argumenterai que l'idée reçue sur la schizophrénie agit comme stéréotype préjudiciable dans la pratique clinique, et donc, qu'elle n'est pas défendable du point de vue pragmatique (en plus de ne pas posséder de justification scientifique). Finalement, dans la troisième partie, je définirai certaines étapes cruciales qui sont nécessaires pour remplacer l'approche dominante dans la compréhension et le traitement actuels des maladies mentales graves.

\section{L'idée reçue concernant la schizophrénie manque de crédibilité scientifique}

La présentation complète de la thèse voulant que l'idée reçue sur la schizophrénie manque de crédibilité scientifique implique un examen détaillé du dossier de recherche (Bentall, 1990 ; Boyle, 2002 ; Heinrichs, 2001) ainsi qu'un examen critique minutieux du programme de recherche associé avec l'idée reçue, avec ses critères de preuve, son potentiel de progrès (Poland, sous presse), ainsi que de l'intégrité de la communauté scientifique engagée dans cette recherche (Poland et Spaulding, à paraître). Même sans une telle évaluation complète, il est instructif de considérer les problèmes de validité, d'hétérogénéité et de définition phénotypique, trois problèmes largement reconnus concernant ce que la plupart des cliniciens et une grande partie de la population croient être vrai à propos de la schizophrénie. Ces trois problèmes suggèrent fortement que l'idée reçue concernant la schizophrénie manque de crédibilité scientifique et, donc, que le concept de schizophrénie est au mieux une façon d'imposer artificiellement un concept au domaine des maladies mentales graves qui a des conséquences potentiellement préjudiciables pour la recherche ainsi que pour les pratiques cliniques et sociales.

Tout d'abord, il n'a jamais vraiment été démontré que le concept de schizophrénie est scientifiquement valide, qu'il s'agisse de la validité du construit 
théorique ou de la validité prédictive. Établir la validité d'un construit scientifique requiert ou bien que les caractéristiques critérielles soient corrélées entre elles (c'est-à-dire, qu'elles constituent un syndrome) ou bien qu'il y ait un cadre théorique ou empirique bien établi qui permette l'élaboration et l'incorporation du concept. Cependant, il n'y a jamais eu suffisamment de preuves scientifiques démontrant que les supposés signes et symptômes de la schizophrénie sont corrélés entre eux (voir Boyle, 2002). Dans le DSM, le fait que l'entrée "schizophrénie " mentionne certaines caractéristiques (vagues, évaluatives, subjectives et disjonctives) comme critères de diagnostic (voir annexe) n'établit pas que ces caractéristiques constituent un véritable syndrome (c'est-à-dire des traits qui ont tendance à se produire simultanément). Et, comme l'ont fait remarquer les critiques (voir Boyle, 2002 ; Kirk et Kutchins, 1992 ; Poland, 2001 et 2002), les critères du DSM pour la schizophrénie ne reposent pas sur une recherche appropriée, convaincante et bien conçue qui établirait que les caractéristiques critérielles sont empiriquement reliées ou que la catégorie (telle que définie par les critères) a toutes autres formes d'intégrité empirique ou théorique. Plutôt, la "validation " des critères a été établie en se basant sur la façon dont ils se conforment aux jugements cliniques d'experts ou à d'autres ensembles de critères diagnostiques ${ }^{7}$.

Malheureusement, la "validation " d'un ensemble spécifique de critères diagnostiques, soit par rapport au " jugement clinique d'un expert ", soit par rapport à d'autres ensembles de critères, est loin d'établir que les critères sont empiriquement corrélés entre eux et qu'ils constituent donc un véritable syndrome. De plus, une telle façon d'aborder la validation est loin d'établir que le concept de schizophrénie a un contenu empirique ou théorique. Étant donné que les critères du DSM concernant la schizophrénie ont été développés dans un contexte "athéorique " dans lequel ni les données empiriques concernant l'étiologie, la pathologie et la réponse au traitement ni les modèles ayant un support empirique n'ont joué aucun rôle dans les décisions à propos des critères diagnostiques, il n'y a pas de raison de croire que les critères du DSM sont suffisants pour distinguer une condition pathologique spécifique ou pour regrouper des individus vraiment semblables du point de vue clinique. Quant au processus de développement du DSM, il n'y a aucune raison de croire que le concept de schizophrénie (défini selon ses critères) est suffisamment élaboré théoriquement ou empiriquement pour établir sa validité. Il n’y a pas non plus de cadres théoriques supportés empiriquement dans lesquels la " schizophrénie » est bien définie (indépendamment du processus de développement du DSM). Le " modèle neurodéveloppemental de la schizophrénie ", à

7. De plus, les critères du DSM pour la schizophrénie ont été développés dans le contexte d'un processus biaisé et politisé, c'est-à-dire que les processus par lesquels le DSM-III et ses descendants (DSM-III-R et DSM-IV) ont été développés ne satisfont pas les standards de base de compétence et de rigueur scientifique (voir, Kirk et Kutchins, 1992 ; Caplan 1995 ; Poland, 2001 et 2002). Conséquemment, il est douteux qu'une seule catégorie du DSM soit basée empiriquement ou théoriquement sur des critères appropriés. 
la mode de nos jours, n'a pas de support empirique, et il est plutôt spéculatif dans son état actuel, comme nous l'accorderont des chercheurs responsables dans ce domaine (voir Hirsch et Weinberger, 2003). En conséquence de ce manque de fondement empirique ou théorique, le concept de schizophrénie ne satisfait pas aux critères de base de la validité des concepts et ne peut donc être considéré comme une construction scientifique bien définie. Ce constat soulève de sérieux doutes quant à la crédibilité scientifique de toute forme de recherche sur la schizophrénie (voir Boyle, 2002, et Poland, sous presse).

Le dossier de la recherche sur la schizophrénie est, de plus, rempli de découvertes négatives, non reproduites, contradictoires, faibles, vagues ou ininterprétables, et qui comportent des lacunes au point de vue méthodologique ${ }^{8}$. De telles découvertes ne fournissent aucun support aux hypothèses scientifiques concernant la schizophrénie, même si elles comprennent des quantités de données qui ont amené certains à se prononcer comme si diverses hypothèses avaient été testées et confirmées. Or, à la lumière d'un tel dossier de recherche et de l'absence de validité du concept mentionné plus haut, il est douteux qu'une hypothèse concernant la schizophrénie ait été testée de façon rigoureuse ou ait déjà reçu quelque support empirique. Notons en outre que le succès prédictif apparent (le fait que la schizophrénie soit héréditaire ou qu'elle réponde à certains genres de traitements) est habituellement obtenu à l'aide de définitions vagues et disjonctives, et de méthodologies de recherche qui ne sont pas conçues pour déterminer si la catégorie a ou non une véritable valeur prédictive. Tout comme les astrologues peuvent acquérir un certain pouvoir prédictif sur le futur à condition que leurs prévisions soient suffisamment disjonctives ou vagues, des "prévisions " concernant le concept de schizophrénie (ou le spectre de la schizophrénie) peuvent sembler avoir une valeur prédictive en raison de leur caractère vague et disjonctif. Ainsi, non seulement la validité du concept proprement dit pose problème, mais la validité prédictive du concept de schizophrénie n'est pas bien établie non plus. D'aucuns pourraient considérer que c'est une hérésie de dire une telle chose, toutefois, il n'y a pas eu d'amélioration substantielle dans les connaissances scientifiques à propos de la schizophrénie dans les cent dernières années?.

Un second problème généralement reconnu concernant l'utilisation de la catégorie diagnostique de la schizophrénie en clinique et en recherche concerne son hétérogénéité quant aux caractéristiques critérielles, aux caractéristiques et processus psychologiques, biologiques et contextuels (par

8. Voir Heinrichs, 2001, pour une revue critique d'un large éventail d'hypothèses de recherche concernant la schizophrénie.

9. Ceux qui sont sous l'emprise de l'idée reçue voient la signification de cette absence de progrès comme un signe de l'immaturité de la science actuelle et de la complexité de la maladie : avec plus de temps et de ressources, la recherche devrait produire une compréhension de la maladie cérébrale nommée "schizophrénie ". Cependant, l'hypothèse de remplacement, à savoir que la schizophrénie n'existe pas et que le concept est scientifiquement insignifiant semble mieux confirmée jusqu'à maintenant par le dossier de recherche que ne le sont l'idée reçue et cette perspective optimiste sur le programme de recherche qui y est associé. 
exemple, sociaux) (Heinrichs, 1993, 2001 ; Andreasen et Carpenter, 1993). Les individus classés comme "schizophrènes " sont susceptibles d'être différents les uns des autres même par rapport aux caractéristiques cliniques qui servent de fondement au diagnostic (c'est-à-dire, les critères du DSM), ainsi que par rapport aux processus biologiques, psychologiques, comportementaux et sociaux qui opèrent à la fois à l'intérieur et à l'extérieur. Une telle hétérogénéité de critères et de processus implique que la dynamique clinique (évolution, résultat, réponse à l'intervention) des conditions spécifiques auxquelles le diagnostic a été appliqué varient beaucoup, comme l'ont remarqué à plusieurs reprises les cliniciens et les chercheurs.

En dépit du fait que cette hétérogénéité soit largement reconnue, il existe un différend substantiel quant à sa signification. Ceux qui sont fortement partisans de l'existence de la schizophrénie suggèrent qu'il y a hétérogénéité parce que la schizophrénie est une maladie unitaire (ou une maladie spectrale relevant de multiples maladies) qui est associée à une grande variété de contextes biologiques, psychologiques ou sociaux avec lesquels elle interagit. Ils croient qu'une telle hétérogénéité deviendra mieux gérée et comprise avec le temps et les progrès de la recherche (voir Andreasen, 2001 ; Green, 2001). Ceux qui ne participent pas à la croyance que "la schizophrénie " se rapporte à un trouble bien défini ${ }^{10}$ suggèrent que l'hétérogénéité fait partie du support empirique démontrant que la catégorie n'a pas de signification sur le plan scientifique et clinique - que la schizophrénie, en fait, n'existe pas. De tels critiques, qui nient l'existence de la schizophrénie (par exemple, Boyle, 2002 ; Bentall, 1990 ; Poland, sous presse), voient la catégorie comme une notion artificielle dans un champ de phénomènes multiples constitué de normes, complexe, dynamique et interactif, lequel requiert un type de conceptualisation radicalement différent pour être compris adéquatement (voir Spaulding et al., 2003).

Tout au moins, on ne devrait pas supposer que l'une ou l'autre de ces interprétations de l'hétérogénéité diagnostique est correcte. Cette question devrait être résolue à l'aide de données scientifiques. Tant qu'elle n'est pas résolue, la crédibilité scientifique de la catégorie diagnostique est, pour le moins, douteuse. Si l'on considère l'absence établie de validité prédictive et de validité du concept de la catégorie même, ce doute est sérieux. En effet, tout ce que nous savons avec certitude, c'est que plusieurs individus ont été regroupés sous l'étiquette "schizophrénie ", que ces individus présentent une hétérogénéité importante

10. Cela ne revient pas à dire qu'il n'y a pas du tout de maladies cérébrales, mais tout simplement qu'à l'heure actuelle un engagement concernant l'idée que la "schizophrénie » correspond à une maladie cérébrale est sans fondement. Il est certainement possible que quelques individus qui s'avèrent tomber dans une catégorie définie par les critères de la schizophrénie selon le DSM-IV ont, parmi plusieurs, dans leur condition, une maladie cérébrale ou autre. Toutefois cela ne justifie aucunement que la catégorie soit censée désigner une maladie cérébrale; dans tous les cas, on doit se rappeler que toute condition ne mène pas à des problèmes qui devraient être considérés comme une maladie. 
quant aux différents aspects de leur fonctionnement, et que, pour le moment, on ne s'entend pas sur ce qui les unit (si unité il y a).

Le doute en ce qui concerne la crédibilité scientifique est finalement renforcé par un autre problème généralement reconnu au sujet de la schizophrénie, celui de la définition phénotypique (c'est-à-dire, à quel trait se rapporte le terme "schizophrénie " ?), un problème qui a émergé récemment avec de plus en plus de clarté dans le contexte de la recherche génétique. Au cours des deux dernières décennies, incités par des déclarations voulant que le dossier de la recherche ait clairement et fortement établi qu'il y a une composante génétique dans l'étiologie de la schizophrénie (voir Gottesman, 1991), des efforts de recherche considérables ont été entrepris en vue de découvrir la base génétique de la schizophrénie à l'aide des méthodologies de la recherche microgénétique (par exemple, l'analyse d'association et de liaison génétique). Jusqu'à présent, ce type de recherche est généralement reconnu comme ayant échoué dans sa tentative d'identification des gènes, de régions de gènes ou de constellations de gènes spécifiques qui seraient soit le siège [locus] d'un trouble génétique, soit des marqueurs pour de tels sièges [loci]. Pour diverses raisons, toutes les supposées découvertes ont été discréditées. À présent, il ne semble pas y avoir de " pistes prometteuses » dans ce domaine de recherche sur la schizophrénie, bien que certains chercheurs enthousiastes affirment souvent le contraire ${ }^{11}$.

L'explication courante de cet échec de la recherche est : $a$ ) que les bases génétiques de la schizophrénie sont plutôt complexes (car plusieurs gènes, interactions entre gènes et avec l'environnement sont en cause) ; et $b$ ) qu'il manque un phénotype bien défini pouvant servir de cible à la recherche micro-génétique. La première composante de l'explication est une répétition des hypothèses génétiques ad hoc qui font suite à l'échec des programmes de recherche d'épidémiologie génétique (études de jumeaux, de familles, et d'adoptions) à découvrir un support empirique pour l'hypothèse d'un gène unique qui ne requiert aucune contribution environnementale. Les chercheurs dans ce domaine, même s'ils ne la formuleraient pas de la façon dont je viens de le faire, reconnaissent que l'hypothèse polygénique et génétique multifactorielle sur la schizophrénie est telle qu'on ne sait pas combien de gènes sont en cause, quelles sont leurs contributions, quelle est la contribution de l'environnement et quels processus de développement sont en jeu. Cela ne constitue pas une hypothèse scientifique impressionnante, pire, elle présuppose sans preuve l'existence de la "véritable schizophrénie".

La seconde composante expliquant l'échec de la recherche microgénétique est la reconnaissance explicite que le concept de schizophrénie n'est pas

11. Voir Harrison et Owen, 2003, et Kennedy et al., 2003. Ces exemples sont typiques du genre de déclarations enthousiastes qui ont été faites pendant des décennies sur la base de supports empiriques limités et dans le contexte de modèles et hypothèses génétiques essentiellement sans contenu (voir ci-dessous). Voir Ploman et McGuffin, 2003, pour une évaluation plus réaliste de l'état de la recherche dans ce domaine. 
tout à fait adéquat pour ce genre de recherche : en effet, pour les raisons mentionnées plus haut, il est trop mal défini. Ce type de recherche requiert des phénotypes bien définis qui peuvent être mesurés avec précision et régularité, et qui présentent un degré suffisant d'homogénéité catégorielle. Jusqu'à ce que de tels phénotypes soient identifiés, la recherche micro-génétique ne pourra avancer ${ }^{12}$. Ni les chercheurs dans ce domaine ni les consommateurs de cette recherche ne semblent être conscients que l'existence d'un phénotype mal défini compromet aussi les études originelles sur la famille, les jumeaux et l'adoption qui supportent le postulat empirique de la recherche micro-génétique (c'est-à-dire, qu'une composante génétique bien établie joue un rôle dans l'étiologie de la schizophrénie). Qu'est-ce que cela signifie exactement que de dire qu'une composante génétique reconnue joue un rôle dans l'étiologie de la schizophrénie si le terme même de "schizophrénie " est mal défini et n'a pas de référent clair ? Dans tous les cas (par exemple, dans les études de familles, de jumeaux, d'adoption, de liaison et d'association génétique), il est tout à fait impossible d'interpréter les découvertes empiriques si la variable critique de la recherche est mal définie. Tout au plus, les corrélations empiriquement relevées, même si elles sont statistiquement significatives, ne font guère plus qu'établir, dans un échantillon donné, une correspondance entre une variable qui nous intéresse (par exemple, les patrons de transmission familiale) et l'étiquette "schizophrénie ». Dans des conditions méthodologiques appropriées, une telle découverte pourrait servir de base à l'établissement d'une corrélation entre la variable et l'étiquette dans une population plus étendue. Cependant, la recherche ne peut pas et ne devrait pas être interprétée au-delà de ces limites ${ }^{13}$.

Donc, à la lumière de ces trois problèmes (validité, hétérogénéité, définition phénotypique) il semblerait que l'idée reçue ne soit rien de plus qu'un ensemble de croyances sans fondement (si elles ont même une signification) à propos d'une supposée maladie appelée "schizophrénie » et des gens hypothétiquement aux prises avec cette " maladie ». Autrement dit l'idée reçue n'est pas scientifiquement crédible. De plus, en conséquence des trois problèmes mentionnés ci-dessus, lorsque des individus sont classifiés comme souffrant de schizophrénie, il y a une perte substantielle d'information (à savoir, les détails spécifiques supportant le « diagnostic ») sans profit compensatoire quant au

12. À ce point, il importe de remettre l'accent sur le fait que les critiques ne nient pas qu'il existe une chose telle que la maladie mentale grave ; l'objet du débat est de savoir si « la schizophrénie " ajoute quoi que ce soit ou bien nuit aux pratiques scientifiques (et cliniques) concernant la maladie mentale grave. Dans le cas de la génétique de la "schizophrénie ", on soutient que l'étiquette est un obstacle sérieux à la recherche.

13. Cela ne signifie pas qu'il ne procède pas à pleine vapeur. Les chercheurs dans ce domaine peinent à identifier un trait suffisamment bien défini et mesurable qui manifeste ne serait-ce qu'une faible association avec la catégorie de diagnostic (mal définie) de la schizophrénie. Des exemples de tels traits sont : des déficits dans «la recherche visuelle » et des anormalités dans les potentiels évoqués à P300 et P50, associées avec le traitement sensoriel des sons (nouveauté, filtrage) Voir Egan et al., 2003, pour une discussion de tels " phénotypes intermédiaires ». 
pouvoir prédictif ou pour la compréhension de la condition de la personne ${ }^{14}$. En réalité, comme nous allons le voir ci-dessous, l'étiquette "schizophrénie " obscurcit la complexité qui doit être prise en considération pour que les objectifs cliniques soient poursuivis avec efficacité. En plus, elle fournit une compréhension simpliste des gens qui sont l'objet de la pratique clinique et subissent le contexte social dans lequel une telle pratique évolue. Conséquemment, le concept de schizophrénie et l'idée reçue à laquelle il est associé fonctionnent comme un stéréotype nuisible qui introduit un risque substantiel de gaspillage, d'erreurs et de dommages dans les contextes cliniques.

\section{L'idée reçue n'a pas d'utilité pragmatique}

Jusqu'à présent nous avons présenté un argument supportant la thèse que l'idée reçue sur la schizophrénie n'est pas scientifiquement crédible. Une approche parallèle pour défendre la légitimité et la pertinence du rôle étendu du concept de schizophrénie et des thèses qui y sont associées implique des arguments pragmatiques selon lesquels la schizophrénie joue un rôle utile dans divers contextes (scientifiques, cliniques, éducatifs ou sociaux, par exemple). Une discussion complète examinerait (entre autres) le rôle de la schizophrénie et de l'idée reçue dans ces arguments : encouragent-ils ou non la recherche scientifique (par exemple, le programme de recherche sur la schizophrénie est-il scientifiquement progressif ?), les valeurs et objectifs des cliniques de santé mentale, de l'éducation, encouragent-ils ou non le bien-être en société tel qu'il est mis de l'avant par l'ordre public et les lois sur la santé mentale ? (voir Poland et Spaulding, à paraître). Dans cette section, je mettrai l'accent sur le rôle de la schizophrénie et de l'idée reçue dans les pratiques cliniques. Je présenterai un argument qui tente de démontrer que l'idée reçue sur la schizophrénie introduit des partis pris préjudiciables dans les pratiques cliniques et, par conséquent, qu'elle va à l'encontre de certaines valeurs et objectifs cliniques essentiels. Une composante clé de la justification pragmatique du maintien du concept de schizophrénie et de l'idée reçue qui y est associée sera donc mise en question.

Dans la pratique clinique qui traite les maladies mentales graves, il y a plusieurs valeurs et objectifs constitutifs :

1. la résolution de l'incertitude clinique concernant les questions suivantes : Quelle information est pertinente et informative ? Quel est le problème de cette personne (si problème il y a) ? Quels processus causaux sont en jeu ? Quelle est la signification de tels problèmes et processus dans la vie d'une personne souffrant d'une maladie mentale grave ? Quelle intervention est susceptible d'être efficace ?

14. En l'absence d'une théorie des processus causaux en jeu dans le développement d'un supposé trait, et sans caractérisation précise de ce trait, il est plutôt problématique d'interpréter causalement l'héritabilité et autres statistiques épidémiologiques (cf., Block, 1996 ; Lewontin, 2000 ; Sober, 2000). 
2. la conception, la mise sur pied et le contrôle des stratégies et techniques d'intervention efficaces visant le traitement, la médication, la palliation ou la réhabilitation ;

3. la conception et la mise sur pied d'infrastructures sociales thérapeutiques éthiquement appropriées pour l'exercice de pratiques cliniques visant à aider des gens qui souffrent de maladies mentales graves.

Si la schizophrénie et l'idée reçue sont considérées comme défendables au point de vue pragmatique, elles devraient pouvoir contribuer à ce que les trois objectifs précédents soient atteints. Cependant, comme nous allons le voir maintenant, un parti pris résultant de l'idée reçue sur la schizophrénie peut survenir au moins dans cinq domaines généraux de la pratique clinique : le traitement de l'information, l'élaboration d'inférences, la compréhension clinique, l'intervention, ainsi que les identités, rôles et relations cliniques (c'est-à-dire, l'infrastructure sociale). Ensemble, de tels partis pris vont à l'encontre des objectifs et des valeurs des pratiques cliniques (c'est-à-dire, 13 ) et nuisent à la fois aux cliniciens et à ceux qui ont besoin de leur aide.

\section{Le traitement de l'information par le clinicien}

Le traitement de l'information par le clinicien comprend entre autres l'observation, l'attention, la recherche d'information, la mémoire et l'enregistrement de l'information. De tels processus déterminent quelle information est accessible pour le raisonnement, le jugement et la prise de décision clinique, et, par conséquent, quelle information joue un rôle dans la formation des émotions, attitudes et motivations du clinicien. Quand le traitement de l'information est influencé par l'idée reçue sur la schizophrénie, le parti pris suivant est encouragé :

1. La tendance à observer, amasser, enregistrer, souligner et se rappeler d'abord d'un ensemble restreint d'informations concernant les caractéristiques pathologiques et cliniquement identifiables (par exemple, les hallucinations, les idées délirantes, les comportements bizarres, désorganisés, ainsi que les « symptômes négatifs » comme le manque de motivation, la diminution des affects, la pauvreté de pensée et de parole).

Dans la pratique diagnostique qui est guidée par le concept de schizophrénie, les types d'information jugés du plus grand intérêt clinique ont tendance à être les caractéristiques cliniques pathologiques énumérées dans les critères du DSM. En plus de rendre de telles caractéristiques encore plus saillantes, cela encourage la catégorisation d'évènements ambigus (par exemple, les actions bizarres) comme instances de ces critères, ainsi que l'effort pour identifier systématiquement leur origine et leur relation dans des dossiers cliniques. Donc, le premier parti pris mène à la création d'un riche corpus d'informations cliniques relatives à un ensemble pauvre et restreint de caractéristiques que le 
clinicien identifie et conçoit facilement comme des indicateurs de pathologie. De telles caractéristiques sont monnaie courante dans les discours des psychiatres sur la schizophrénie et sont recherchées dans des entrevues de diagnostic clinique dyadiques, soulignées dans des dossiers cliniques et considérées suffisantes pour un diagnostic.

L'attention est donc portée sur ces caractéristiques au détriment d'un ensemble plus vaste de caractéristiques qui ne sont pas incluses dans les critères du DSM et qu'on ne daigne pas considérer comme signe diagnostique primaire. Ainsi, plusieurs types d'informations ne sont pas examinés ou recueillis du tout, ils sont même relégués à un statut secondaire ou non pertinent pour les besoins de l'évaluation diagnostique : par exemple, l'information concernant le fonctionnement de la personne sur plusieurs plans, biologique, psychologique, comportemental et social ${ }^{15}$; l'information sur le fonctionnement spécifique dans un grand nombre de contextes sociaux et personnels, le point de vue de la personne sur l'histoire de sa vie, ses projets, ses plans, ses perspectives et ses relations. Ceux qui sont sous l'emprise de l'idée reçue ont tendance à minimiser l'importance de ce genre d'information lorsque vient le temps de découvrir ce qui est problématique chez la personne. Ou bien ce genre d'information n'est pas recueillie du tout, ou bien elle est recueillie mais sa signification première est d'aider à découvrir un diagnostic en accord avec les critères du DSM. Cela s'applique autant à la collecte du clinicien sur l'historique médical et social de la personne qu'à l'utilisation d'examens psychologiques et d'évaluations de capacités. Par exemple, ce type d'information secondaire est souvent utilisé pour corroborer un diagnostic clinique, utilisant par exemple des questions comme : Quelle est l'historique diagnostique de la personne ? Quel est l'historique familial pour ce qui est de la maladie mentale ? Les résultats des examens psychologiques se conformentils au diagnostic de schizophrénie ? Y a-t-il eu un déclin récent dans le fonctionnement ? Y a-t-il une détérioration cliniquement significative dans le fonctionnement? Ainsi, même quand on recueille de l'information sur la schizophrénie autre que celle qui concerne les caractéristiques présentes selon les critères du DSM, la façon avec laquelle opère l'idée reçue a tendance à minimiser son rôle dans le diagnostic et à la placer dans un cadre orienté vers la pathologie, lequel est élaboré autour de l'étiquette diagnostique.

Pourquoi les conséquences du parti pris 1 sont-elles préjudiciables ? La constitution d'une base de donnée restreinte orientée vers la pathologie ne permet pas au clinicien d'être suffisamment informé pour comprendre le problème et le genre de processus causaux en jeu dans la vie de la personne ou dans le milieu clinique. Cette base de données n'offre pas aux cliniciens

15. Par exemple, si une personne rapporte des hallucinations angoissantes et dérangeantes, un clinicien peut prescrire une drogue anti-psychotique réputée être efficace pour aider à maitriser les hallucinations. Cependant, un diagnostic de schizophrénie n'est pas requis pour prédire que la drogue peut être efficace et n'ajoute rien à la compréhension de ce qui se passe dans un tel cas. 
l'information requise pour gérer l'hétérogénéité considérable de problèmes et de processus que l'on retrouve chez les individus souffrant de maladies mentales graves, et donc, ne leur donne pas l'information nécessaire pour résoudre l'ambiguité clinique. En plus, un corpus d'information appauvri et orienté vers la pathologie [pathology-oriented] ne fournit pas la base pour une compréhension adéquate de la personne, de sa vie, de ses objectifs et ses valeurs. L'impression que quelque chose pose problème chez la personne domine l'activité clinique au lieu d'être une composante possible de son état. Le résultat est que la personne dont la vie est au centre de la pratique clinique perd son statut en plus de perdre d'avantage de pouvoir (comme nous le verrons plus bas). Cela est alarmant, étant donné que le point de vue et l'action de la personne sont parmi les facteurs les plus importants du succès de la pratique clinique.

\section{Les pratiques inférentielles}

L'idée reçue sur la schizophrénie mène à des pratiques inférentielles qui manifestent le parti pris suivant :

2. La tendance à inférer l'existence d'un processus inhérent à la maladie qui sert à expliquer la présence - ainsi que les relations entre des caractéristiques cliniques et à interpréter des évènements et les caractéristiques de la personne comme manifestations de l'état d'une maladie biologique individuelle.

Lorsque l'information jugée pertinente pour le diagnostic est évaluée, le clinicien sous l'emprise de l'idée reçue est conditionné à voir des groupes de caractéristiques et d'évènements comme étant interreliés et comme des manifestations d'une seule maladie sous-jacente. De plus, des caractéristiques et des évènements essentiellement ambigus (par exemple, un témoignage mentionnant une hallucination, une parole délirante, un acte bizarre) sont susceptibles d'être interprétés comme des manifestations d'une maladie cérébrale ${ }^{16}$.

Bien que certains défenseurs du stéréotype de la schizophrénie avouent que la schizophrénie n'est pas une maladie unitaire, mais plutôt une " maladie spectrale » qui varie selon un nombre de facteurs, ou bien de " multiples maladies " (c'est-à-dire que le terme "schizophrénie » est appliqué à divers processus morbides chez différents individus), on suppose invariablement que, dans les cas individuels, certains processus de la maladie causent les manifestations

16. Il existe au moins cinq types d'analyse utiles et appropriés pour comprendre le fonctionnement, les problèmes et les processus en jeu dans la maladie mentale grave : les plans neuropsychologique (par exemple, la régulation $\mathrm{SNC}$ ), neurocognitif (p. ex., l'attention, le contrôle exécutif, la mémoire), sociocognitif (p. ex. la résolution de problème, l'inférence causale, les attributions, la connaissance de soi et des autres), sociocomportemental (p. ex., l'activation comportementale ; les habilités dans divers domaines de fonctionnement, la régulation psychophysiologique de l'émotion, l'humeur, l'impulsion), et socioenvironnementaux (p. ex., les relations, la conformité ou la déviance, le statut social, l'environnement de vie). Voir Spaulding et al., (2003) pour une discussion de ces types d'analyse. 
cliniques. Or il n'y a pas de bonnes données scientifiques sur ce que pourraient être les processus de la maladie (même si la spéculation abonde) ${ }^{17}$ ou sur la façon dont la maladie pourrait être liée causalement à ses supposées manifestations cliniques. Les cliniciens qui expriment le parti pris 2 ont tendance à ignorer ou minimiser l'importance des hypothèses causales qui n'impliquent pas une maladie cérébrale de base. Par exemple, de tels cliniciens ont tendance à ignorer les hypothèses à propos de l'impact environnemental direct ou les boucles de rétroaction entre l'environnement et l'individu, les interactions biologiques, psychologiques et sociales complexes, ainsi que les processus psychologiques « normaux » qui concernent le choix, l'apprentissage social et les processus attributionnels (voir Corrigan et Penn, 2001, pour des travaux représentatifs dans ce domaine). Étant donné que les caractéristiques et les évènements individuels qui obtiennent l'attention des cliniciens sont très possiblement le résultat de tels autres types de processus causaux, une tendance rigide et stéréotypique à inférer des hypothèses concernant la présence d'une maladie peut conduire à l'occultation des véritables causes (qui tiennent, en certains cas, de l'identification à un rôle appris ou d'un processus psychologique normal), et favoriser un parti pris sévère et coûteux pour une compréhension "individualiste " des problèmes, c'est-à-dire, la conception qu'il sont inhérents à la personne (par exemple, le résultat d'une pathologie du cerveau $)^{18}$.

Le second parti pris mène aussi les cliniciens à ignorer la possibilité que les caractéristiques cliniques observées soient indépendantes les unes des autres (par exemple, que les hallucinations d'une personne soient liées à un dérèglement biochimique tandis que ses paroles délirantes reflètent un historique complexe d'apprentissage social), ou qu'elles soient interreliées d'une autre façon (par exemple, une escalade d'excitation provoquant des difficultés chez une personne dont les capacités à gérer un conflit social angoissant sont déjà amoindries), deux possibilités dont l'existence est régulièrement

17. Le rapport d'une hallucination ou de la prononciation de paroles délirantes (entre autres) est essentiellement ambigu parce que chacun peut être le reflet d'un certain nombre d'autres processus causaux. Par exemple, bien que les hallucinations soient de vrais phénomènes, les rapports d'hallucinations ne sont pas tous véridiques et même si des paroles délirantes sont souvent prononcées, elles peuvent avoir différentes sortes de signification clinique allant des supposées dérégulations pathophysiologiques jusqu'aux historiques complexes d'apprentissage social, aux processus psychologiques normaux et carrément aux manipulations.

18. À noter que le postulat de processus causaux à la base des divers signes et symboles supposés de la schizophrénie ne réussit pas à identifier le processus pathologique de la schizophrénie pour deux raisons. Le statut de maladie pour ces processus n'est pas établi par un tel postulat. Et l'existence de processus associés avec les caractéristiques prises individuellement n'établit pas qu'un processus morbide est associé à l'étiquette diagnostique, étant donné que les caractéristiques en jeu ne sont pas spécifiques à la "schizophrénie » et ne sont pas présentes dans tous les cas de schizophrénie. Tout le monde devrait être impliqué dans le développement d'une compréhension causale de la maladie mentale grave, mais l'émergence d'une telle compréhension causale ne valide pas directement un modèle de maladie pour ce qui est de la schizophrénie. 
confirmée $^{19}$. Ainsi, une tendance rigide et stéréotypique à avancer des hypothèses qui accordent un rôle central à une maladie a tendance à favoriser diverses erreurs dans les pratiques d'évaluation clinique et, comme nous allons le voir maintenant, ces erreurs contribuent à leur tour à miner l'intervention et la compréhension des cliniciens.

\section{La compréhension des cliniciens}

La compréhension des cliniciens affectés par l'idée reçue sur la schizophrénie mène au parti pris suivant :

3. La tendance à voir les gens classifiés comme schizophrènes selon le modèle de cette maladie (dans laquelle un processus morbide de base module la perception, la pensée, l'émotion et le comportement), et, donc, à les voir comme des victimes d'une maladie cérébrale sur laquelle ils n'ont aucun contrôle.

Comme nous venons d'en discuter, des pratiques inférentielles biaisées par l'influence de l'idée reçue sur la schizophrénie favorisent une compréhension simpliste des causes de l'état de la personne, résultant d'un processus pathologique de base ainsi que des cascades pathogéniques auxquelles mène ce processus : toutes les caractéristiques pathologiques sont comprises comme les conséquences de causes qui sont en aval du processus de base. Dans un tel cadre d'interprétation, on considère que la perception, la pensée, l'émotion et le comportement de la personne sont modulés par un processus pathologique interne et donc ceux-ci sont plutôt vus comme l'effet causal psychologiquement insignifiant d'un cerveau malade. Les hypothèses causales mentionnées plus haut (c'est-à-dire, celles qui concernent des problèmes indépendants, des causes environnementales, des boucles de rétroaction interactive complexes et des processus psychologiques normaux) sont plutôt ignorées ou minimisées. Ainsi, une vision orientée vers la pathologie de la personne, laquelle est vue comme la victime passive d'une maladie cérébrale, a tendance à prédominer. Le stéréotype de la schizophrénie compromet donc la pratique

19. Une conséquence particulièrement importante du parti pris en cause ici est une tendance à "décontextualiser » les problèmes de la personne. Le stéréotype de la schizophrénie implique qu'un processus morbide de base existe dans l'individu et que : a) il est peut-être partiellement causé par des facteurs d'ordre environnemental ; b) il peut avoir des conséquences dans un contexte environnemental ; mais, c) ni les antécédents causaux, ni les conséquences causales de la maladie, ni n'importe quelle autre caractéristique du contexte ne font partie du processus même de la maladie. C'est-à-dire que la compréhension d'un trouble chez la personne peut complètement exclure le contexte. Voir Spaulding, Sullivan, et Poland, 2003, pour une discussion de la raison pour laquelle une vision décontextualisée de la maladie mentale grave mène à un sérieux malentendu concernant la nature des problèmes d'une personne et de la raison pour laquelle privilégier une cause unique à des processus morbides dans le cerveau d'un individu est erroné (car la causalité peut bouger dans toutes les directions, elle peut impliquer des caractéristiques et des processus appartenant à n'importe quel type d'analyse causale, des processus "normaux " aussi bien que pathologiques). 
clinique et fausse les impressions des cliniciens quant au caractère et à la complexité des circonstances dans lesquelles les individus évoluent et quant aux problèmes auxquels ils tentent de faire face.

Le troisième parti pris favorise plutôt une compréhension simpliste des gens (et non seulement du réseau causal dont il font partie) qui souffrent de maladie mentale grave. Voir la personne comme la victime passive d'une maladie cérébrale diminue considérablement notre compréhension aussi bien du point de vue de la personne que de son fonctionnement. Si un clinicien a tendance à concevoir la perception, la pensée, l'émotion et le comportement d'une personne comme étant essentiellement l'effet causal psychologiquement insignifiant d'un cerveau malade, alors le point de vue et les actions de la personne seront mis de côté, puisqu'il sont conçus comme des manifestations du processus de base de cerveau, et donc, comme des choses à être éradiquées par le traitement plutôt que comme des composantes essentielles de la compréhension. Un tel refus de prendre en considération la personne et son fonctionnement entraîne le clinicien à perdre de vue des choses telles que :

- la façon dont la personne comprend ce qui lui arrive dans un contexte clinique;

- la réaction de la personne au fait d'être classée et traitée de certaines façons par des cliniciens et par d'autres personnes;

- les rôles sociaux que la personne occupe, ou les manières par lesquelles une personne pourrait résister aux pressions pour qu'elle occupe de tels rôles (par exemple, le rôle d'un " patient souffrant d'un trouble mental »);

— le point de vue de la personne sur sa vie : ses valeurs, ses objectifs, ses aspirations, ses projets, ses perspectives, sa compréhension historique et sa compréhension de sa propre situation actuelle;

- la manière dont l'architecture cognitive ainsi que les processus psychologiques et sociaux normaux opèrent en ce qui concerne le comportement ;

— la manière dont les actions de la personne peuvent être légitimées à la lumière de sa situation, de ses limitations et de la façon dont elle est traitée.

Sans une telle compréhension, une dimension importante de la personne sera inintelligible au clinicien, surtout compte tenu des limitations présentes, si l'on n'observe qu'un ensemble restreint de facteurs et qu'on suppose qu'un processus cérébral fondamental cause le problème. Sans une véritable évaluation de la condition de la personne de son propre point de vue, il est difficile d'établir des relations thérapeutiques efficaces et respectueuses. Cela crée des obstacles supplémentaires pour le genre de compréhension qui devrait être à la base des pratiques d'évaluation et d'intervention cliniques. 


\section{Les pratiques d'intervention}

Dans toute bonne pratique clinique, il n'y a pas de place pour une base de donnée incomplète ou orientée vers la pathologie, pour des inférences biaisées ou pour une compréhension simpliste. Malheureusement, ces préjugés, conjointement avec diverses composantes de l'idée reçue sur la schizophrénie, entraînent en pratique clinique un quatrième parti pris quant à la conception et à la mise en œuvre des interventions :

4. La tendance à concevoir la supposée maladie cérébrale et ses manifestations comme étant des cibles primaires d'intervention et à utiliser l'observation des symptômes comme mesure principale du succès ou de l'échec du traitement.

Dans le cadre d'interprétation inspirée par l'idée reçue, le statut de maladie cérébrale attribué à la condition de la personne occupe une position centrale, et toute intervention vise à contrôler et à gérer la maladie. Pour ce faire, la thérapie qui utilise des médicaments psychotropiques est la forme d'intervention la plus importante, tandis que le contrôle des symptômes est la forme la plus importante d'évaluation du succès ou de l'échec du traitement. Il résulte de ce parti pris que la grande variété d'autres processus causaux possibles que nous avons mentionnés plus haut aura tendance à rester non identifiée et à ne pas être prise en considération. Le $4^{\mathrm{e}}$ parti pris est dangereux parce qu'il a tendance à court-circuiter toute observation clinique sérieuse à propos de l'intervention, ce qui entraîne des plans d'intervention routiniers et mal conçus, laissant le clinicien sans ressources adéquates pour comprendre pourquoi une intervention choisie n'est pas efficace (par exemple, l'absence de réponse à la médication $)^{20}$.

\section{Les rôles cliniques, les identités et les relations}

Le dernier domaine dans lequel l'idée reçue sur la schizophrénie engendre un parti pris est l'infrastructure sociale des pratiques et des processus cliniques (la façon dont les identités, les rôles et les relations thérapeutiques prennent forme).

5. La tendance à créer une infrastructure sociale pour l'activité clinique dans laquelle l'identité de la personne devient celle de victime d'une maladie sur laquelle elle n'a aucun contrôle. Le rôle de la personne est celui d'un patient dont la responsabilité première est d'obéir aux traitements prescrits par le clinicien et les rapports de la personne relèvent d'un statut d'autorité inférieur aux autres.

Les cliniciens sous l'influence de l'idée reçue sur la schizophrénie seront portés à interagir avec les personnes étiquetées comme schizophrènes comme

20. Voir Spaulding, Sullivan et Poland (2003) pour une approche clinique des maladies mentales graves qui fournit des analyses causales précises. 
si elles étaient des victimes passives d'un processus pathologique qui doit être traité par un médecin, lequel utilise des stratégies et techniques essentiellement biomédicales. L'aspect particulièrement critique de cette intervention, c'est que de tels cliniciens auront fortement tendance à " éduquer » la personne sous leur soin à adopter la même vision qu'eux-mêmes : on apprend aux personnes étiquetées comme schizophrènes à croire qu'elles sont des victimes passives d'une maladie cérébrale nommée "schizophrénie ". Ainsi, ces personnes sont amenées à internaliser un stéréotype social stigmatisant ("la schizophrénie ») et à s'attribuer des caractéristiques (par exemple, négative, interne, stable, globale) susceptibles d'avoir des conséquences personnelles préjudiciables (voir Kinderman, 2001) ${ }^{21}$.

Les cliniciens sous l'influence de l'idée reçue sur la schizophrénie auront aussi tendance à éduquer de la même façon tout un chacun, incluant la famille et les amis de la personne et les autres membres du personnel de l'hôpital2 ${ }^{22}$. La création d'une interprétation partagée par tous les partis concernés mène à une structuration rigide du monde social de la personne, dans laquelle son identité à la première et à la troisième personne est celle d'une victime passive d'une maladie cérébrale, dont les caractéristiques et le comportement pathologiques occupent l'attention de façon prédominante. Par conséquent, le statut, les rôles et les rapports de la personne dans son monde social sont influencés négativement. Plus précisément, aidée par l'apprentissage implicite et explicite, et de puissants processus sociaux, cette forme d'éducation tend à renforcer les rôles et l'identité du patient souffrant d'un trouble mental dysfonctionnel au lieu de créer des occasions de trouver des solutions importantes (voir Spaulding, et al. 2003).

Finalement, l'impact général du cinquième parti pris et les pratiques éducationnelles particulières qu'il engendre comprend de nombreuses autres conséquences que nous devons mentionner ici : la dépréciation involontaire de la personne, l'encouragement d'une attitude passive face à ses problèmes, la perte supplémentaire de pouvoir dans la vie, la diminution de l'engagement de la personne dans son propre traitement, la création d'une relation thérapeutique viciée. En conséquence, le cinquième parti pris favorise le développement de processus sociaux et cliniques préjudiciables et improductifs.

\section{Le résultat des partis pris}

Les cinq partis pris mentionnés ci-dessus qui ont cours dans les pratiques cliniques pour traiter les maladies mentales graves démontre clairement comment l'idée reçue sur la schizophrénie, au lieu de contribuer à la mise sur pied de

21. Voir Spaulding et al., (2003) pour une présentation systématique d'un cadre destiné au développement de plans d'intervention complexes et calibrés individuellement.

22. Bien que sérieusement erronées pour les raisons mentionnées dans le texte, ces pratiques d'éducation du patient sont souvent bien intentionnées : elles reflètent une préoccupation clinique concernant la personne qui subit le traitement et elles visent souvent l'introduction d'un cadre d'interprétation cognitif très utile pour elle. 
services cliniques essentiels ainsi qu'à la poursuite de valeurs et objectifs cliniques importants, met en péril la pratique clinique de multiples façons ; de ce fait, elle est préjudiciable et inutile. Ainsi, il n'y a pas d'argument pragmatique plausible pour défendre l'existence ou le maintien du concept de schizophrénie et de l'idée reçue qui lui est associée, et des pratiques cliniques qui en résultent. Tout argument de ce genre est remis en cause par l'effet préjudiciable de l'approche ainsi que par l'existence d'autres approches dont l'efficacité supérieure a été démontrée (voir Spaulding et al., 2003).

\section{Quatre étapes vers un monde sans schizophrénie}

Le fait que l'idée reçue sur la schizophrénie manque à la fois de crédibilité scientifique et d'utilité pragmatique signifie qu'une réforme des pratiques cliniques, sociales et de la recherche actuelle pour le traitement des maladies mentales graves est fortement suggérée. Or c'est une chose d'argumenter, comme de nombreux critiques l'ont fait (par exemple, Szasz, 1988 ; Sarbin, 1990 ; Boyle, 2002), que l'état des choses actuel ne devrait pas exister parce qu'il manque de crédibilité scientifique, qu'il est inutile, préjudiciable et éthiquement indéfendable. C'en est une autre d'identifier et d'emprunter un chemin clair et praticable vers un état de choses plus souhaitable, ce qu'aucun critique ne semble avoir été capable de faire.

Nous proposons une heuristique pour penser à la recherche de stratégies de réforme, et celles-ci peuvent être divisées en quatre étapes majeures :

Étape 1 : établir visiblement et de manière persuasive que le cadre actuel de la conceptualisation et de l'organisation des pratiques concernant le domaine des maladies mentales graves ne peut être défendu et ne devrait pas exister.

Étape 2 : fournir un cadre de rechange dans lequel il est possible de poursuivre les divers objectifs qui sont trop mal servis par le cadre actuel, et le rendre plausible.

Étape 3 : fournir une analyse adéquate de l'emprise du cadre actuel et une stratégie révolutionnaire pour renverser cette emprise.

Étape 4 : ajouter à la stratégie révolutionnaire une stratégie pour gérer l'instabilité qui sera inévitablement précipitée par l'étape 1 et par les transitions envisagées et poursuivies aux étapes 2 et 3.

Certains diront sans doute que chacune de ces étapes doit être poursuivie efficacement pour que les efforts de réforme réussissent. Quelques-unes des raisons possibles expliquant pourquoi les critiques précédentes n'ont pas été efficaces pour déstabiliser et encore moins pour remplacer l'idée reçue sont : 1) que les arguments offerts contre celle-ci n'ont pas été suffisamment convaincants ou suffisamment diffusés pour être entendus par des auditoires importants ; 2) qu'un autre cadre de pensée viable n'a pas été proposé et rendu plausible ; 3) qu'aucune proposition suffisamment consistante et plausible n'a été faite, et encore moins poursuivie, pour expliquer comment contrer l'emprise massive de l'idée reçue ; et 4) qu'aucun plan clair concernant la façon 
de gérer efficacement une transition de l'idée reçue vers une approche de remplacement plus souhaitable n'a été offert ${ }^{23}$. Dans ce qui suit, je vais faire quelques remarques sur les sortes de propositions et actions requises pour la mobilisation d'un effort de réforme sérieux.

En ce qui concerne l'étape 1, les première et deuxième parties du présent texte ont été consacrées à souligner les sortes d'arguments qui peuvent être mobilisés pour démontrer que l'idée reçue sur la schizophrénie n'est ni scientifiquement crédible ni pragmatiquement utile. Convenablement élaborés et augmentés, de tels arguments pourraient bien être suffisants pour démontrer de façon crédible pourquoi l'idée reçue n'est en réalité pas défendable scientifiquement et pragmatiquement. Cependant, pour être des composantes efficaces d'un effort de réforme, de tels arguments doivent être très publicisés de façon à être en mesure de rejoindre les divers auditoires qui ont des enjeux dans cette réforme. Poursuivi efficacement, l'effet d'un tel assaut sur le caractère défendable de l'idée reçue sera inévitablement d'ébranler la confiance en celle-ci ainsi que les pratiques qui s'y rapportent. Donc, l'effet sera quelque peu déstabilisant en ce sens que les pratiques actuelles pourraient de plus en plus être remises en question et critiquées, que des groupes et des individus pourraient commencer à chercher des solutions de remplacement, que les défenseurs des pratiques actuelles augmenteront leurs efforts défensifs (par exemple, en augmentant les efforts d'éducation publique et en essayant de discréditer les critiques) ; ainsi, idéalement, il deviendra plus fréquent parmi ceux qui possèdent le pouvoir politique de parler des critiques et de la nécessité de la réforme. Minimalement, un examen critique plus rigoureux des pratiques actuelles sera effectué. L'étape 1 crée donc l'occasion de changer en éveillant la conscience des partis intéressés, en déstabilisant les pratiques actuelles, en jetant de la lumière sur des problèmes qui doivent sérieusement être analysés, et en stimulant l'implantation d'examens rigoureux et de processus de réforme des pratiques actuelles.

L'étape 2 propose la condition essentielle aux efforts de réformes réalistes dans n'importe quel domaine de pratique scientifique ou clinique : c'està-dire, le développement d'une pratique de rechange et la démonstration explicite et visible de sa plausibilité2 ${ }^{24}$. Dans la situation actuelle, le fardeau est uniquement sur les épaules des critiques, qui, en plus de relever et de souligner les problèmes concernant le concept de schizophrénie et l'idée reçue afférente, se doivent d'élaborer et de soutenir une autre approche crédible pour la compréhension de la maladie mentale grave, qui garantira la recherche productive et les pratiques cliniques ainsi que d'autres pratiques sociales efficaces ${ }^{25}$.

23. Du point de vue du clinicien guidé par l'idée reçue, la constitution de rapports appropriés basés sur une telle compréhension partagée de la personne est cruciale pour la mise sur pied de plans d'intervention clinique efficaces ainsi que pour aider le patient dans ses efforts à gérer sa maladie. Cependant, ce point de vue repose sur plusieurs hypothèses douteuses.

24. Mary Boyle (2002) est celle qui est la plus près de traiter au moins ces diverses préoccupations.

25. Voir Friedman (1999) pour une discussion instructive de la façon dont cette exigence a opéré dans l'histoire de la physique. 
Cette condition est importante parce que les critiques avancées à l'étape 1 sont essentiellement déstabilisantes (par exemple, elles encouragent une perte de confiance en l'idée reçue ainsi qu'un questionnement, et la recherche de solution de rechange à celle-ci). Les individus, groupes et institutions dépendants de l'idée reçue d'une façon ou d'une autre (voir plus bas) ont besoin d'une autre solution pour pouvoir l'abandonner : sans solution de rechange crédible et viable en main, les critiques (peu importe à quel point elles sont percutantes) seront incapables de susciter une réforme et mourront dans l'œuf ${ }^{26}$.

L'étape 3, l'analyse et l'abandon de l'emprise profonde de l'idée reçue, est requise si les efforts de réforme visant à remplacer l'idée reçue en faveur d'une solution de rechange viable sont socialement et politiquement possibles, et même efficaces. Comme la discussion au début de ce texte l'a établi, l'idée reçue sur la schizophrénie est implantée dans de nombreuses institutions et pratiques sociales, et elle sert une variété d'intérêts et d'objectifs : n'importe lequel de ces éléments, et peut-être tous, pourraient contribuer à une forte résistance au changement qui est perçu comme étant préjudiciable et perturbateur. D'une part, l'idée reçue est profondément liée aux droits acquis des associations professionnelles (p. ex., en psychiatrie, en psychologie clinique ou en travail social), des entités commerciales (entre autres, les compagnies pharmaceutiques et d'assurance), des groupes de protection du consommateur (p. ex., l'Alliance nationale pour les malades mentaux) et des institutions sociales (comme l'assurance-maladie financée publiquement). Tout projet de réforme subira fortement l'impact des pressions provenant de ces intérêts. D'autre part, l'idée reçue est aussi puissamment informée et maintenue par des idéologies et valeurs culturelles qui s'incarnent dans des cadres visant à " médicaliser " les problèmes de vie et à répondre à de tels problèmes avec une efficacité technologique toujours croissante. À un public qui exige des diagnostics médicaux formels et des solutions rapides et efficaces à ses problèmes s'ajoute un corps professionnel et d'autres institutions organisées pour la satisfaction de ces demandes (comme les assureurs publics et privés, les cliniques de santé mentale) et spécialisées en cette matière.

L'effet de la force d'inertie d'une telle emprise est augmenté par le degré avec lequel l'idée reçue forme les consciences publique et professionnelle en ce qui a trait aux maladies mentales graves. Une combinaison d'efforts

26. Notons qu'il ne suffit pas simplement d'élaborer un cadre de remplacement pour la conceptualisation et les pratiques en jeu dans la maladie mentale grave. Pour établir la plausibilité de telles solutions, divers critères de crédibilité et de viabilité doivent aussi être visiblement présents. Par exemple, une autre conceptualisation du domaine de la maladie mentale grave doit clairement être supérieure aux approches actuelles en ce qui concerne : 1) son degré d'efficacité par rapport aux demandes imposées par les différentes caractéristiques du domaine (par exemple, l'ambiguité, une grande variation dans les multiples dimensions de fonctionnement, la complexité causale et dynamique, l'importance des aspects normatifs et de la perspective sur la maladie mentale) ; 2) le degré d'intégration du cadre aux sciences changeantes concernées par le fonctionnement humain ; et 3) le degré de support empirique pour le succès du cadre dans la pratique clinique et scientifique. 
" éducationnels » agressifs et une réceptivité non critique à de tels efforts a substantiellement renforcé l'emprise de l'idée reçue (par exemple, les messages transmis par la publicité directe quant à la médication psychiatrique et intégrés dans l'éducation continue des médecins à propos des maladies mentales et leur traitement). De plus, bien qu'il soit possible d'entendre des voix critiques et très préoccupées par ces questions, il y a de plus en plus de preuves que la communauté scientifique, dont on s'attend à ce qu'elle fonctionne comme régulatrice des croyances et des pratiques défendables, est elle-même en train d'éprouver des soupçons. Ils concernent d'une part la qualité des standards qui opèrent dans les sous-communautés scientifiques et d'autre part les vérifications très incomplètes des conflits d'intérêts et autres sources de menaces individuelles et communautaires à l'objectivité scientifique. De telles absences de standards rigoureux et de tels compromis à l'objectivité révèlent une absence significative de discours critique à l'intérieur même des communautés scientifiques dont nous dépendons et qui jouissent d'une autorité intellectuelle considérable (par exemple, les activités de recherche fondamentale, le processus d'examen par les pairs, l'attribution de bourses, l'organisation de conférences et la supervision gouvernementale ont toutes été compromises par de mauvais standards et par l'absence d'objectivité $)^{27}$.

À la lumière de cet aperçu des diverses composantes de l'emprise de l'idée reçue, l'étape 3 d'une stratégie de réforme pourrait paraître plutôt inquiétante. En effet, il est manifeste que les efforts de réforme devront inévitablement être compréhensifs et soutenus sur une longue période de temps. Toute proposition sérieuse pour se défaire de l'emprise de l'idée reçue sur la schizophrénie aura besoin d'encourager toute activité reliée à l'éducation formelle et informelle, l'augmentation de la mise sur pied d'examens critiques rigoureux et la prise de responsabilité dans l'activité clinique et scientifique, la reconstitution des communautés scientifiques appropriées, l'amélioration des pratiques de levée de fonds et enfin la réorganisation de la supervision administrative et des restrictions éthiques sur les conflits d'intérêts.

Dans une stratégie de réforme, l'étape 4 concerne la gestion de l'instabilité qui sera inévitablement précipitée par l'étape 1, ainsi que les transitions envisagées et poursuivies aux étapes 2 et $3^{28}$. En bref, pour ce qui est de la compréhension et des formes de pratiques, les transitions auront inévitablement un effet négatif sur certains des intérêts et objectifs d'individus, de groupes et d'institutions qui dépendent de la situation actuelle. Comme l'a démontré la discussion sur l'emprise de l'idée reçue, plusieurs formes de cette dépendance ont déjà été créées et entretenues au cours des dernières décennies. Il est également évident que des changements radicaux dans la compréhension et les formes de pratique peuvent éventuellement perturber de telles

27. Voir Poland et Spaulding, à paraître.

28. En effet, l'importance du genre de stratégie de gestion requise à cette étape, une stratégie qui (entre autres) minimise les torts infligés à ceux qui dépendent des arrangements actuels, a à peine été reconnue par les critiques précédents de l'idée reçue. 
relations de dépendance et de compromettre les intérêts et objectifs connexes. Par exemple, les relations thérapeutiques dans lesquelles la continuité des soins est d'une importance vitale devraient être protégées. Étant donné que l'accès aux soins de santé est rendu possible par remboursement d'assurances pour des services liés à des conditions spécifiques cliniquement définies, le processus de remplacement de la conceptualisation de maladie mentale grave en termes de catégories diagnostiques rudimentaires par des spécifications de problèmes plus détaillées et scientifiquement défendables devrait être poursuivi de façon à n'abandonner personne et à ne pas empêcher ceux qui en ont besoin d'avoir accès au soutien et aux services. D'autres facteurs sont en jeu tels que le statut d'emploi des cliniciens et autres travailleurs en santé mentale, le statut et la carrière des chercheurs et le destin de divers programmes de recherche. Conséquemment, un fardeau majeur pour tous ceux concernés par la réalisation de la réforme des pratiques courantes consiste à identifier et à minimiser le potentiel de dommages collatéraux qui pourraient résulter du remplacement d'une forme de compréhension et de pratique par une autre. Ainsi, la tâche de se défaire de l'emprise de l'idée reçue et celle d'installer d'autres formes de compréhension et de pratique sont compliquées par cette contrainte éthique et sociale de minimiser les dommages collatéraux.

Les quatre étapes que nous venons de discuter sont les composantes essentielles d'une stratégie de réforme des pratiques scientifiques, cliniques et sociales actuelles associées à la maladie mentale grave. La question de savoir si la maladie mentale est mieux conceptualisée, étudiée et approchée (cliniquement et socialement) dans le langage de l'idée reçue — informée comme elle l'est par le concept de schizophrénie -, ou si la maladie mentale grave est mieux conceptualisée, étudiée, et approchée dans le langage d'un autre cadre de pensée tel qu'il a été suggéré dans les parties 1 et 2 de ce texte est avant tout une question de crédibilité scientifique et d'utilité pragmatique. Cependant, savoir si de telles questions de crédibilité et d'utilité peuvent être étudiées efficacement à l'intérieur des communautés scientifiques présentement constituées et si les efforts de réforme, une fois lancés, peuvent être poursuivis efficacement sont des questions sociales d'ordre politique et éthique dont la compréhension et la résolution requièrent des outils très différents. Ainsi, les frontières traditionnelles entre les sujets scientifiques et sociaux deviennent floues, si elles ne sont pas carrément remises en question ${ }^{29}$.

(Traduction de Jean-Charles Pelland)

\section{Références}

American Psychiatric Association, Diagnostic and Statistical Manual of Mental Disorders, 4th Edition (DSM-IV), Washington, American Psychiatric Association Press, 1994.

29. Voir Longino (1990, 2002), Kitcher (2001) et Hacking (1999) pour d'importantes discussions concernant la façon de conceptualiser les relations entre les considérations et sujets scientifiques, et les considérations et sujets sociaux. 
Andreasen, N. Brave New Brain, New York, Oxford University Press, 2001.

Andreasen, N. et W. Carpenter. "Diagnosis and Classification of Schizophrenia ", Schizophrenia Bulletin, 19, 1993, p. 199-214.

Bentall, R. dir., Reconstructing Schizophrenia, New York, Routledge, 1990.

Block, N. « How Heritability Misleads About Race ", Boston Review, XX, n 6, 1996, p. 30-35.

Boyle, M. «Schizophrenia : a Scientific Delusion ?, $2^{\text {nd }}$ Edition ». New York, Routledge, 2002.

Corrigan, P. et D. Penn, dir. "Social Cognition and Schizophrenia », Washington, American Psychological Association Press, 2001.

Egan, M., M. Leboyer, et D. Weinberger. "Intermediate Phenotypes in Genetic Studies of Schizophrenia », dans S. Hirsch et D. Weinberger, dir., Schizophrenia, Massachusetts, Blackwell Science, 2003.

Friedman, M. Dynamics of Reason, Stanford, CSLI Publications, 1999.

Gottesman, I. Schizophrenia Genesis : the Origins of Madness, New York, Freeman, 1991.

—. "Schizophrenia Epigenesist : Past, Present, and Future ", Acta Psychiatrica Scandinavica, 90, 1994, p. 26-33.

Green, M. Schizophrenia Revealed, New York, Norton, 2001.

Hacking, I. The Social Construction of What? ", Cambridge, Harvard University Press, 1999.

Heinrichs, R.W. « Schizophrenia and the Brain : Conditions for a Neuropsychology of Madness ", American Psychologist, 48, 1993, p. 221-233.

-. In Search of Madness, New York, Oxford University Press, 2001.

Hirsch, S. et D. Weinberger, dir., Schizophrenia, Oxford, Blackwell Science, 2003.

Kinderman, P. "Changing Causal Attributions ", dans P. Corrigan et D. Penn, dir., Social Cognition and Schizophrenia, Washington, D.C., American Psychological Association, 2001, p. 195-215.

Kirk, S. et H. Kutchins. The Selling of the DSM : the Rhetoric of Science in Psychiatry, New York, Aldine De Gruyter, 1992.

Kitcher, P. Science, Truth, and Democracy, New York, Oxford University Press, 2001.

Laing, R. The Politics of Experience, New York, Pantheon Books, 1967.

Lewontin, R. The Triple Helix, Cambridge, Mass., Harvard University Press, 2000.

Longino, H. Science as Social Knowledge, Princeton, Princeton University Press, 1990.

- The Fate of Knowledge, Princeton, Princeton University Press, 2002.

Mcevoy, J.P., P.L Scheifler, et A. Frances, « Treatment of Schizophrenia », The Journal of Clinical Psychiatry, 60, Supplement 11, 1999.

Mouldin, S. et I. Gottesman, "Genes, Experience, and Chance in Schizophrenia : Positioning for the $21^{\text {st }}$ Century ", Schizophrenia Bulletin, 23, 1997, p. 547-561.

Ploman, R. et P. Mcguffin. "Psychopathology in the Post - Genomic Era ", Annual Review of Psychology, 54, 2003, p. 205-228.

Poland, J. "Review of the DSM Sourcebook, Volume 1. Metapsychology ", [en ligne : http ://Mentalhelp.Net/Books/Books.Php ?Type=De\&Id=557, 2001].

—. "Review of the DSM Sourcebook, Volume 2. Metapsychology », [en ligne:

http ://Mentalhelp.Net/Books/Books.Php ?Type=De\&Id=996, 2002]. 
- "How to Move Beyond the Concept of Schizophrenia ", dans M. Chung, W. Fulford et G. Graham, dir., The Philosophical Understanding of Schizophrenia, Oxford, Oxford University Press, sous presse.

Poland, J. et W. Spaulding. Crisis and Revolution : Toward a Reconceptualization of Psychopathology, Cambridge, Mass., MIT Press, à paraître.

Sarbin, T. « Toward the Obsolescence of the Schizophrenia Hypothesis », The Journal of Mind and Behaviour, 11, 1990, p. 259-284.

Scheff, T. Being Mentally Ill : a Sociological Theory, $3^{\text {rd }}$ Edition, New York, Aldine De Gruyter, 1999.

Sober, E. "The Meaning of Genetic Causation ", dans A. Buchanan, D. Brock, N. Daniels et D. Winkler, From Chance To Choice, Cambridge, Cambridge University Press, 2000.

Spaulding, W., M. Sullivan, et J. Poland, Treatment and Rehabilitation of Severe Mental Illness. New York, Guilford Press, 2003.

Szasz, T. Schizophrenia : the Sacred Symbol of Psychiatry, Syracuse, Syracuse University Press, 1988.

Wakefield, J. « The Concept of Mental Disorder : on the Boundary Between Biological and Social Values ", American Psychologist, 47, 1992, p. 373-388.

\section{Annexe}

Critères du DSM-IV pour la schizophrénie

A. Symptômes caractéristiques : Deux (ou plus) des manifestations suivantes sont présentes, chacune pendant une portion significative d'une période d'un mois (ou moins quand elles répondent favorablement au traitement) :

1) idées délirantes

2) hallucinations

3) discours désorganisé (c.-à-d., coq-à-l'âne fréquents ou incohérence)

4) comportement grossièrement désorganisé ou catatonique

5) symptômes négatifs, par ex., émoussement affectif, alogie ou perte de volonté

N.B. : Un seul symptôme du critère A est requis si les idées délirantes sont bizarres ou si les hallucinations consistent en une voix commentant en permanence le comportement ou les pensées du sujet, ou si, dans les hallucinations, plusieurs voix conversent entre elles.

B. Dysfonctionnement social ou des activités : Pendant une grande portion de temps depuis le début de la perturbation, un ou plusieurs domaines majeurs du fonctionnement tels que le travail, les relations interpersonnelles, ou les soins personnels sont nettement inférieurs à ce qu'il était avant le début de la perturbation (ou, si le trouble a débuté dans l'enfance ou l'adolescence, incapacité à atteindre le niveau de réalisation auquel on aurait pu s'attendre sur le plan relationnel, scolaire, ou dans d'autres activités).

C. Durée : Des signes permanents de la perturbation persistent pendant au moins six mois. Cette période de six mois doit comprendre au moins un mois 
de symptômes (ou moins quand les personnes réagissent favorablement au traitement) qui répondent au critère $\mathrm{A}$ (c.-à-d., symptômes de la phase active) et peut comprendre des périodes de symptômes prodromiques ou résiduels. Pendant ces périodes, les signes de la perturbation peuvent se manifester uniquement par des symptômes négatifs, ou par deux ou plus des symptômes figurant dans le critère A, présents sous une forme atténuée (p. ex., croyances bizarres, perceptions inhabituelles).

D. Exclusion d'un trouble schizo-affectif ou d'un trouble de l'humeur: Le trouble schizo-affectif et le trouble de l'humeur avec caractéristiques psychotiques ont été éliminés, soit parce qu'aucun épisode dépressif majeur, maniaque ou mixte n'a été présent simultanément aux symptômes de la phase active ; soit parce que des épisodes thymiques ont pu être présents pendant les symptômes de la phase active et que leur durée totale a été brève par rapport à la durée des périodes actives et résiduelles.

E. Exclusion d'une affection médicale générale ou due à une substance: la perturbation n'est pas due aux effets physiologiques directs d'une substance (c.-à-d., une drogue donnant lieu à des abus, un médicament) ou d'une affection médicale générale.

F. Relation avec un trouble envahissant du développement : en cas d'antécédent de trouble autistique ou d'un autre trouble envahissant du développement, le diagnostic additionnel de schizophrénie n'est posé que si les idées délirantes ou des hallucinations éprouvées sont également présentes pendant un mois (ou moins quand elles diminuent substantiellement avec le traitement). 